\title{
Donald Smiley Prize
}

The Donald Smiley Prize, established in honour of Donald V. Smiley (1921-1990), internationally renowned scholar of Canadian politics and a former President of the Canadian Political Science Association, will be awarded in 2000 . The prize, to which is attached a monetary award in the amount of $\$ 1,000$, is awarded to the author or authors of the best book published in English or French in a field relating to the study of government and politics in Canada in the preceding two-year period. The award-winning book may be single-authored or multiauthored and will be selected by the Board of Directors of the Canadian Political Science Association, upon the recommendation of the Prize Jury. Textbooks, edited texts or collections of essays will not be considered. The prize is sponsored by McGill-Queen's University Press. Individuals who have published a book in 1998 or 1999 which is eligible for the prize should ensure that their publishers forward one copy of the book by December 10,1999, to each of: (1) Donald Smiley Prize Jury, Canadian Political Science Association, Suite 204, 260 Dalhousie Street, Ottawa, Ontario KIN 7E4; (2) Donald Smiley Prize Jury, Professor Samuel V. LaSelva, Department of Political Science, University of British Columbia, \#C472-1866 Main Mall, Vancouver, British Columbia V6T 1Z1; (3) Donald Smiley Prize Jury, Professor Caroline Andrew, Department of Political Science, University of Ottawa, Ottawa, Ontario K1N 6N5; and (4) Donald Smiley Prize Jury, Professor Andrew F. Cooper, Department of Political Science, University of Waterloo, Waterloo, Ontario N2L 3G1.

\section{Prix Donald-Smiley}

Le Prix Donald-Smiley a été créé en hommage à Donald V. Smiley (1921-1990), grand spécialiste de la politique canadienne dont la réputation dépassait nos frontières et un ancien président de l'Association canadienne de science politique. L'Association décernera le Prix Donald-Smiley, d'une valeur de $1000 \$$, en 2000 à l'auteur, l'auteure ou aux auteurs du meilleur livre publié en français ou en anglais sur un sujet traitant de la politique ou du gouvernement au Canada au cours de la période correspondante de deux ans. Le Conseil d'administration de l'Association désignera le lauréat, la lauréate ou les lauréats à la suite d'une recommandation du Jury du Prix Donald-Smiley. Les manuels scolaires, les livres publiés sous la direction d'une personne ou d'une équipe et les collections d'essais ne seront pas admissibles. Le prix est subventionné par McGill-Queen's University Press. Les personnes qui auraient publié un ouvrage en 1998 ou en 1999 selon les conditions du concours devront, pour être éligibles, demander à leurs éditeurs de 